\title{
Overexpression of miR-21 promotes neural stem cell proliferation and neural differentiation via the Wnt/ $\beta$-catenin signaling pathway in vitro
}

\author{
WEI-MIN ZHANG ${ }^{1}$, ZHI-REN ZHANG ${ }^{2}$, XI-TAO YANG ${ }^{3}$, YONG-GANG ZHANG ${ }^{1}$ and YAN-SHENG GAO ${ }^{1}$ \\ ${ }^{1}$ Department of Neurosurgery; ${ }^{2}$ Medical Department, Zhumadian Central Hospital, Zhumadian, Henan 463000; \\ ${ }^{3}$ Department of Interventional Radiotherapy, Shanghai Ninth People's Hospital, Shanghai Jiao Tong University \\ School of Medicine, Shanghai 200011, P.R. China
}

Received December 11, 2015; Accepted January 20, 2017

DOI: $10.3892 / \mathrm{mmr} .2017 .7856$

\begin{abstract}
The primary aim of the present study was to examine the effects of microRNA-21 (miR-21) on the proliferation and differentiation of rat primary neural stem cells (NSCs) in vitro. miR-21 was overexpressed in NSCs by transfection with a miR-21 mimic. The effects of miR-21 overexpression on NSC proliferation were revealed by Cell Counting kit 8 and 5-ethynyl-2'-deoxyuridine incorporation assay, and miR-21 overexpression was revealed to increase NSC proliferation. miR-21 overexpression was confirmed using reverse transcription-quantitative polymerase chain reaction (RT-qPCR). mRNA and protein expression levels of key molecules ( $\beta$-catenin, cyclin D1, p21 and miR-21) in the Wnt/ $\beta$-catenin signaling pathway were studied by RT-qPCR and western blot analysis. RT-qPCR and western blot analyses revealed that miR-21 overexpression increased $\beta$-catenin and cyclin D1 expression, and decreased p21 expression. These results suggested that miR-21-induced increase in proliferation was mediated by activation of the Wnt/ $\beta$-catenin signaling pathway, since overexpression of miR-21 increased $\beta$-catenin and cyclin D1 expression and reduced p21 expression. Furthermore, inhibition of the Wnt/ $\beta$-catenin pathway with FH535 attenuated the influence of miR-21 overexpression on NSC proliferation, indicating that the factors activated by miR-21 overexpression were
\end{abstract}

Correspondence to: Professor Zhi-Ren Zhang, Medical Department, Zhumadian Central Hospital, 747 Zhonghua Road, Zhumadian, Henan 463000, P.R. China

E-mail: chgn3619@163.com

Professor Xi-Tao Yang, Department of Interventional Radiotherapy, Shanghai Ninth People's Hospital, Shanghai Jiao Tong University School of Medicine, 639 Zhizaoju Road, Shanghai 200011, P.R. China

E-mail: xitao123456@126.com

Key words: microRNA-21, neural stem cells, Wnt/ $\beta$-catenin signaling pathway, proliferation, differentiation inhibited by FH535 treatment. Furthermore, overexpression of miR-21 enhanced the differentiation of NSCs into neurons and inhibited their differentiation into astrocytes. The present study indicated that in primary rat NSCs, overexpression of miR-21 may promote proliferation and differentiation into neurons via the Wnt/ $\beta$-catenin signaling pathway in vitro.

\section{Introduction}

Neural stem cells (NSCs) are present in the central nervous system (CNS) of adults and developing mammals $(1,2)$. They are self-renewing, multipotent and undifferentiated precursor cells that have the ability to differentiate into glial and neuronal lineages. NSCs may be useful in cell-based replacement therapies for neurological disorders, including Alzheimer's disease, traumatic brain injury, spinal cord injuries and retinal disease (3-5). However, poor proliferation and insufficient differentiation of the transplanted NSCs into neurons limit the practical use of NSC-based therapies $(6,7)$, making further study necessary to realize the potential of NSCs in clinical treatment (8-10). Therefore, it is important to explore the molecular and cellular mechanisms that regulate NSC proliferation and differentiation.

The Wnt/ $\beta$-catenin pathway serves an important role in regulating NSC fate. Activation of the Wnt/ $\beta$-catenin pathway promotes NSC proliferation and differentiation $(9,10)$. Hence the requirement for investigating the regulation of the Wnt/ $\beta$-catenin pathway.

MicroRNAs (miRNAs) are small noncoding RNAs $\sim 22 \mathrm{nt}$ in length, which have been demonstrated to be involved in the gene regulation process (11-13). miRNAs are involved in various biological functions, including cell differentiation, proliferation and apoptosis (14-17). miRNAs are involved in stem cell fate and self-renewal, and regulate the expression of stem cell genes $(15,18,19)$.

In the present study, overexpression of miR-21 was demonstrated to enhance the proliferation of rat NSCs and their differentiation into neurons, and to reduce their differentiation into astrocytes via the $\mathrm{Wnt} / \beta$-catenin signaling pathway. 


\section{Materials and methods}

Cell culture and transfection. All procedures requiring the use of laboratory animals were approved and monitored by the Animal Care Committee of Zhumadian Central Hospital (Zhumadian, China). Isolation, culture and identification of NSCs was performed as previously described (20). Pregnant Sprague-Dawley rats (day E14 fetal; 200-250 g; n=6) were obtained from the Shanghai Branch of National Rodent Laboratory Animal Resources (Shanghai, China) and were housed in a controlled $22 \pm 1^{\circ} \mathrm{C}$ environment under $14 / 10 \mathrm{~h}$ light/dark cycles with free access to food and water. Following anesthesia with $50 \mathrm{mg} / \mathrm{kg}$ of ketamine and $10 \mathrm{mg} / \mathrm{kg}$ of xylazine administered intraperitoneally, NSCs were isolated from the hippocampus of 40 embryonic day 14 fetal rats. The tissues were transferred to cold phosphate buffered saline (PBS), minced, and dissociated. The isolated NSCs were cultured in Dulbecco's modified Eagle's medium-F12 medium (Sigma-Aldrich; Merck KGaA, Darmstadt, Germany) supplemented with $1 \% \mathrm{~N}_{2}, 2 \%$ B-27 supplement, 2 mmol/1 glutamine, $20 \mathrm{ng} / \mathrm{ml}$ epidermal growth factor (EGF) and $20 \mathrm{ng} / \mathrm{ml}$ basic fibroblast growth factor (bFGF; Peprotech, Inc. Rocky Hill, NJ, USA). Primary neurospheres were digested with $0.25 \%$ trypsin. EGF and bFGF were removed from the growth medium, and the medium was supplemented with $1 \%$ fetal bovine serum (FBS; Irvine Scientific, Santa Ana, CA, USA). Cells were placed on poly-lysine-coated coverslips in 24-well plates in prior to fixation. miR-21 mimic and scramble were purchased from Thermo Fisher Scientific, Inc. (Waltham, MA, USA). The transfection of miR-21 mimics and scramble miRNA (20 ng/ml) was performed using Lipofectamine 2000 (Invitrogen; Thermo Fisher Scientific, Inc.) according to the manufacturer's protocol. NSCs were incubated with $1 \mu \mathrm{M}$ of the Wnt/ $\beta$-catenin pathway inhibitor FH535 (EMD Millipore, Billerica, MA, USA) for $24 \mathrm{~h}$, on day 7 post-transfection. miR-21 mimic and scramble sequences were: miR-21 mimic: 5'-UAGCUUAUCAGACUGAUGUUGA-3'; miR-21 scramble: 5'-UUCUCCGAACGUGUCACGUTT-3'.

Immunocytochemistry. Cells were fixed with paraformaldehyde (4\%) for $\sim 30 \mathrm{~min}$ at room temperature and permeabilized with $0.2 \%$ Triton $\mathrm{X}-100$. Following blocking with $10 \%$ goat serum, cells were incubated with primary anti- $\beta$-tubulin III (AB15708A4; 1:200; Merck KGaA, Darmstadt, Germany), anti-glial fibrillary acidic protein (GFAP; 04-1062; 1:1,000; Merck KGaA) and anti-nestin antibodies (ab134017; 1:100; Abcam, Cambridge, UK), at $4^{\circ} \mathrm{C}$ overnight. Subsequently, cells were incubated with tetramethylrhodamine isothiocyanate-conjugated goat polyclonal anti-rabbit immunoglobulin (Ig)G (ab145472; 1:300; Abcam), fluorescein isothiocyanate (FITC)-conjugated goat anti-rabbit IgG (bs-0519R-FITC; 1:100; BIOSS, Beijing, China), or FITC-conjugated goat anti-mouse IgG (bs-2511R-FITC; 1:100; BIOSS) for $3 \mathrm{~h}$ at room temperature. Nuclei were stained with DAPI. Coverslips were mounted and slides were analyzed by fluorescence microscopy (Zeiss GmbH, Jena, Germany).

Cell proliferation. NSC proliferation was assessed using Cell Counting kit 8 (CCK8) assay (Dojindo Molecular Technologies, Inc., Kumamoto, Japan) according to the manufacturer's protocol. Proliferation rates were analyzed $0,1,3,5$ and 7 days following transfection. The optical density was measured at a wavelength of $450 \mathrm{~nm}$. In addition, a 5-ethynyl-2'-deoxyuridine (EdU) incorporation assay was used to assess cell proliferation. The single cell suspensions of NSCs from the neurospheres (7 days following transfection) were incubated with the proliferation marker EdU $(0.2 \mathrm{mM} / \mathrm{l})$ for $30 \mathrm{~min}$ at $37^{\circ} \mathrm{C}$. Following incubation, the dissociated cells were seeded onto $100 \mu \mathrm{g} / \mathrm{ml}$ poly-L-lysine-coated coverslips and were stained for the NSC marker nestin using the Click-iT EdU Imaging kit (Thermo Fisher Scientific, Inc.) according to the manufacturer's protocol. Cells were incubated with a rabbit anti-nestin polyclonal primary antibody (ab134017; 1:200; Abcam) for $36 \mathrm{~h}$ at $4^{\circ} \mathrm{C}$, and a Cy3-conjugated goat anti-rabbit IgG secondary antibody (bs-2511R-Cy3; 1:100; BIOSS) for $3 \mathrm{~h}$ at room temperature.

Reverse transcription-quantitative polymerase chain reaction $(R T-q P C R)$. Total RNA was isolated from the cells using TRIzol ${ }^{\circledR}$ reagent (Invitrogen; Thermo Fisher Scientific, Inc.). RNA samples were purified and had optical density 260/280 ratios between 1.8 and 2.0, as confirmed using a Nanodrop Spectrophotometer (Thermo Fisher Scientific, Inc., Wilmington, DE, USA). The extracted RNA was reverse transcribed into cDNA using a RevertAid First Strand cDNA Synthesis kit (Fermentas; Thermo Fisher Scientific, Inc.). For each sample $2 \mu \mathrm{g}$ RNA was used to synthesize the cDNA. The RT-qPCR reaction was conducted using iQ SYBR-Green Supermix (Bio-Rad Laboratories, Inc., Hercules, CA, USA) and regulated by the spectrofluorometric iQ5 Thermal iCycler (Bio-Rad Laboratories, Inc.). qPCR detected the expression of miRNA-21 and mRNA using the SYBR-Green PCR kit (Qiagen, Inc., Valencia, CA, USA) on a 7500 Real-Time PCR system (Applied Biosystems; Thermo Fisher Scientific, Inc.). Amplification was performed under the following conditions: Initial 1 cycle at $95^{\circ} \mathrm{C}$ for $8 \mathrm{~min}$, followed by 42 cycles at $95^{\circ} \mathrm{C}$ for $10 \mathrm{sec}$, at $60^{\circ} \mathrm{C}$ for $40 \mathrm{sec}$ and at $72^{\circ} \mathrm{C}$ for $1 \mathrm{sec}$, and a final extension step at $72^{\circ} \mathrm{C}$ for $10 \mathrm{~min}$. The relative mRNA expression of each gene was calculated using the $2^{-\Delta \Delta \mathrm{Cq}}$ method and normalized to $\beta$-actin (21). The primers used were as follows: miR-21 forward, 5'-CCAGTGAAGATGTGTTCAGCT-3' and reverse, 5'-GCACAGCCAGTAGAAGTAGAT-3'; $\beta$-catenin forward, 5'-GACCACAAGCAGAGTGCTGA-3' and reverse, 5'-ACTCGGGTCTGTCAGGTGAG-3'; cyclin D1 forward, 5'-TGGAGCCCCTGAAGAAGAG-3' and reverse, 5'-AAG TGCGTTGTGCGGTAGC-3'; p21 forward, 5'-CTGCTCTCC CTTCCTCAGAC-3' and reverse, 5'-TGAGGTAGGACCAGG AAACC-3'; $\beta$-tubulin III forward, 5'-AGCAAGGTGCGT GAGGAGTA-3' and reverse, 5'-AAGCCGGGCATGAAG AAGT-3'; GFAP forward, 5'-CAACGTTAAGCTAGCCCT GGACAT-3' and reverse, 5'-CTCACCATCCCGCATCTC CACAGT-3'; and $\beta$-actin forward, 5'-CCCGCGAGTACAACC TTCT-3' and reverse, 5'-CGTCATCCATGGCGAACT-3'.

Western blot analysis. Cells were collected and washed twice with PBS. Cells were lysed using lysis buffer containing $10 \mathrm{mM}$ Tris- $\mathrm{HCl}$ (pH 7.4), 150 mM NaCl, 1\% NP-40, 0.1\% sodium dodecyl sulfate, $1 \%$ sodium deoxycholate and protease inhibitor cocktail tablet (Roche Diagnostics, Basel, Switzerland) for $30 \mathrm{~min}$ at $37^{\circ} \mathrm{C}$. The protein content in each sample was 
A

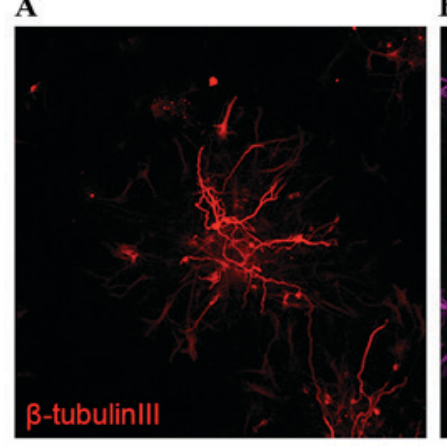

B

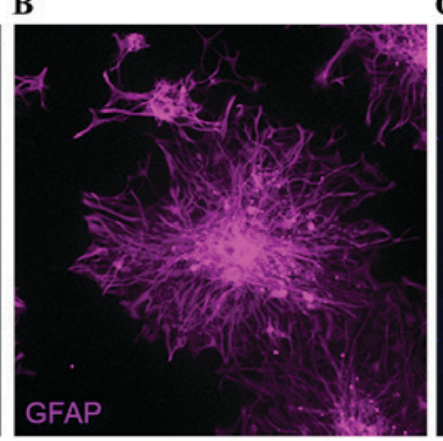

C

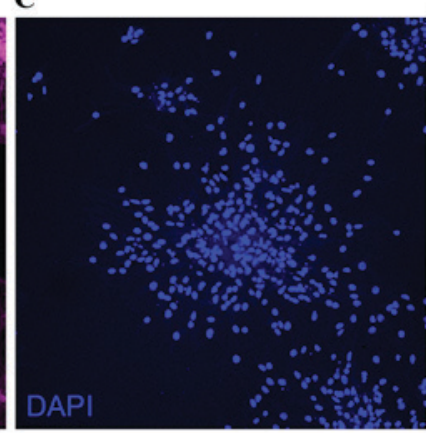

D

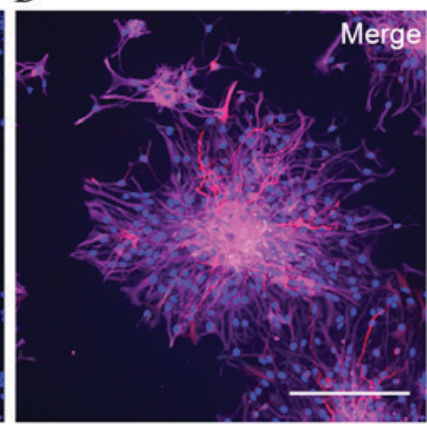

Figure 1. Identification of rat neural stem cells. Cultured cells were induced to differentiate into (A) neurons ( $\beta$-tubulin III-positive cells) and (B) astrocytes (GFAP-positive cells). (C) Nuclei were stained with DAPI and (D) images were merged. Scale bar, $100 \mu \mathrm{m}$. GFAP, glial fibrillary acidic protein.

determined using a Bio-Rad Protein assay kit (Bio-Rad Laboratories, Inc., Hercules, CA, USA). Equal amounts of extracted protein samples $(50 \mu \mathrm{g})$ were separated by $12 \%$ SDS-PAGE and transferred onto a polyvinylidene fluoride membrane, which was blocked with $5 \%$ milk overnight at $4^{\circ} \mathrm{C}$. The membrane was incubated with the following primary antibodies: $\beta$-catenin (Ab4176; 1:2,000), cyclin D1 (ab134175; 1:100), p21 (ab109199; 1:500), $\beta$-tubulin III (AB15708A4; 1:1,000), GFAP (04-1062; 1:2,000) and $\beta$-actin (Santa Cruz Biotechnology, Inc. Ab3700; 1:500) overnight at $37^{\circ} \mathrm{C}$. Subsequently, the membrane was probed with horseradish peroxidase-conjugated goat anti-rabbit IgG (cat no. sc-2004; Santa Cruz Biotechnology, Inc., Dallas, TX, USA) and goat anti-mouse IgG (cat no. sc-2005; Santa Cruz Biotechnology, Inc.) secondary antibodies at 1:1,000 and room temperature for $1 \mathrm{~h}$. Protein bands were visualized using the Beyotime ECL Plus detection kit (Beyotime Institute of Biotechnology, Haimen, China). The band intensities were subsequently quantified using Quantity One software (version 4.62; Bio-Rad Laboratories, Inc.) and normalized to $\beta$-actin as an internal standard.

Statistical analysis. All data are presented as the mean \pm standard deviation of three independent experiments. Statistical analyses were performed using SPSS software version 20.0 (IBM SPSS, Armonk, NY, USA). An unpaired Student's t-test or one-way followed by Fisher's least square difference (LSD) post hoc test was used for a statistical comparison of the mean values between two or four groups, respectively. $\mathrm{P}<0.05$ was considered to indicate a statistically significant difference.

\section{Results}

Identification of rat NSCs. The rat NSCs formed neurospheres and differentiated into neurons and astrocytes 3 days following removal of bFGF (Fig. 1). In addition, the rat NSCs expressed the NSC marker nestin (Fig. 2A), which identified the isolated cells as rat NSCs.

miR-21 promotes rat NSC proliferation. The EdU incorporation assay evaluated the effects of miR-21 on rat NSC proliferation. Cells were treated with $\mathrm{EdU}(0.2 \mathrm{mM} / \mathrm{l})$ for $30 \mathrm{~min}$ on day 7 post-transfection, and the incorporated EdU was analyzed.
The proportion of EdU-positive cells was enhanced $\sim 2$-fold in the miR-21 mimic group (45.72 $\pm 5.39 \%)$ compared with the scramble group $(23.61 \pm 2.65 \%$; $\mathrm{P}<0.05$; Fig. $2 \mathrm{~B})$.

The CCK8 assay was also performed to determine the involvement of miR-21 in the proliferation of transfected rat NSCs. The NSCs in the miR-21 mimic group exhibited faster growth and proliferation rates compared with the scramble group 3, 5, 7 and 10 days post-transfection ( $\mathrm{P}<0.05$; Fig. 2C), indicating that overexpression of miR-21 enhanced rat NSC proliferation.

Overexpression of $m i R-21$ promotes rat NSC proliferation through activation of the Wnt/ $\beta$-catenin signaling pathway. To determine whether the Wnt/ $\beta$-catenin pathway was involved in the miR-21 overexpression-induced increase of rat NSC proliferation, the mRNA and protein expression levels of several associated genes, including $\beta$-catenin, cyclin D1 and p21 were detected by RT-qPCR and western blotting. Overexpression of miR-21 significantly increased $\beta$-catenin and cyclin D1 mRNA expression levels compared with the scramble control $(\mathrm{P}<0.05$; Fig. $3 \mathrm{~A})$, and reduced $\mathrm{p} 21 \mathrm{mRNA}$ expression levels compared with the scramble control $(\mathrm{P}<0.05$; Fig. 3A). $\beta$-catenin and cyclin D1 protein expression levels were also increased in the miR-21 mimic group compared with the scramble control group $(\mathrm{P}<0.05$; Fig. 3B), whereas p21 protein expression levels were decreased $(\mathrm{P}<0.05$; Fig. 3B). These findings suggested that the $\mathrm{Wnt} / \beta$-catenin pathway may be involved in mediating the effects of miR-21 overexpression on rat NSC proliferation.

Suppression of the Wnt/ $\beta$-catenin signaling pathway inhibits the increased rat NSC proliferation induced by overexpression of miR-21. To confirm involvement of the $\mathrm{Wnt} / \beta$-catenin pathway in NSC proliferation, a pharmacological inhibitor, the $\beta$-catenin/T cell factor (TCF) inhibitor (FH535), was used. Treatment with FH535 inhibited miR-21 mimic-induced rat NSC proliferation compared with the scramble control $(\mathrm{P}<0.05$; Fig. 4) and cells transfected with the miR-21 mimic only $(\mathrm{P}<0.05$; Fig. 4$)$.

miR-21 promotes rat NSC differentiation to neurons and inhibits differentiation to astrocytes. miR-21 promoted the differentiation of rat NSCs to neurons and inhibited the differentiation to astrocytes, as confirmed by increased 

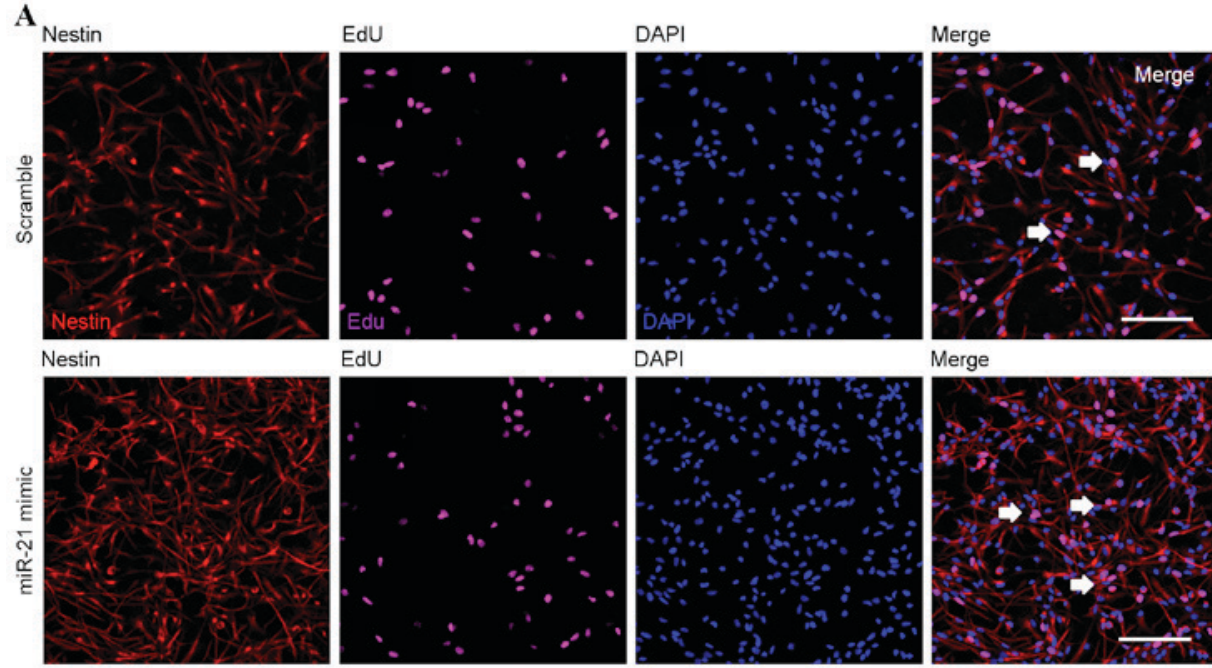

DAPI

Merge
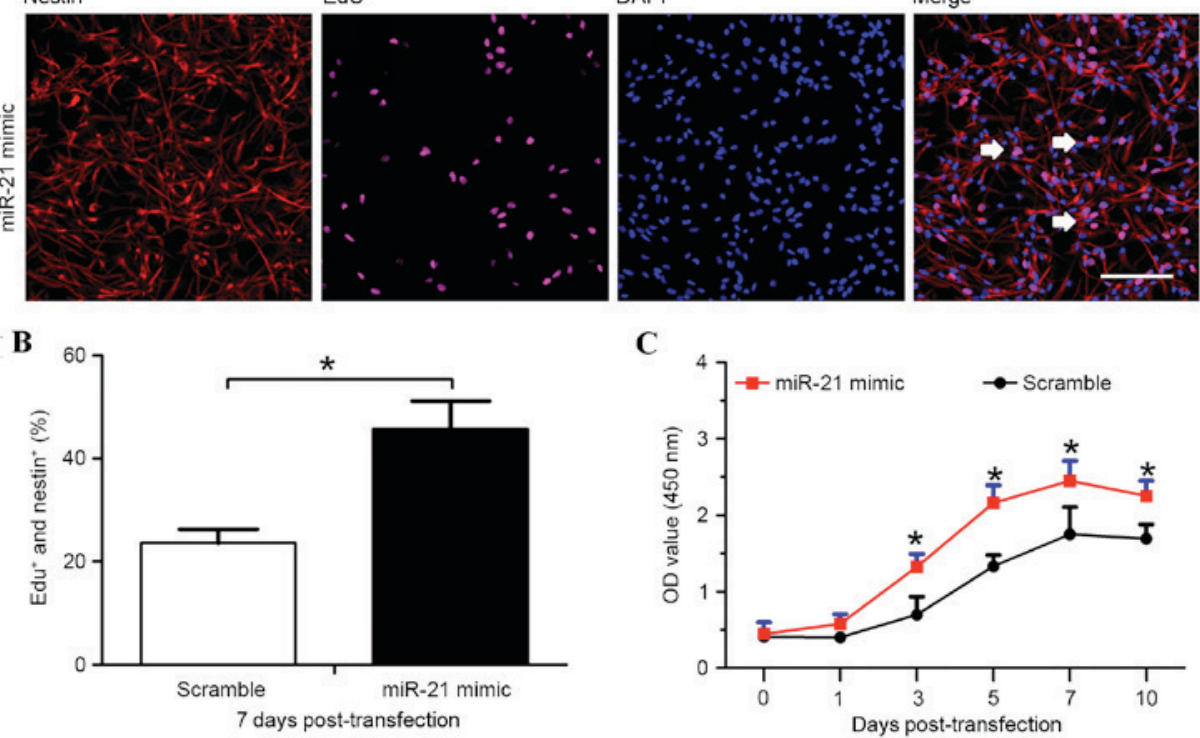

Figure 2. Effects of miR-21 overexpression on rat neural stem cell proliferation. (A) EdU incorporation assay revealed a visibly increased proportion of EdU-positive cells in the miR-21 mimic group. White arrows indicate merged EdU-positive cells. Scale bar, $100 \mu \mathrm{m}$. (B) The number of EdU-positive cells was quantified. "P<0.05 vs. scramble control group. (C) Results of the Cell Counting kit 8 assay. "P<0.05 vs. scramble control group. Data are expressed as the mean \pm standard deviation $(n=3)$. EdU, 5-ethynyl-2'-deoxyuridine; miR-21, microRNA-21; OD, optical density.
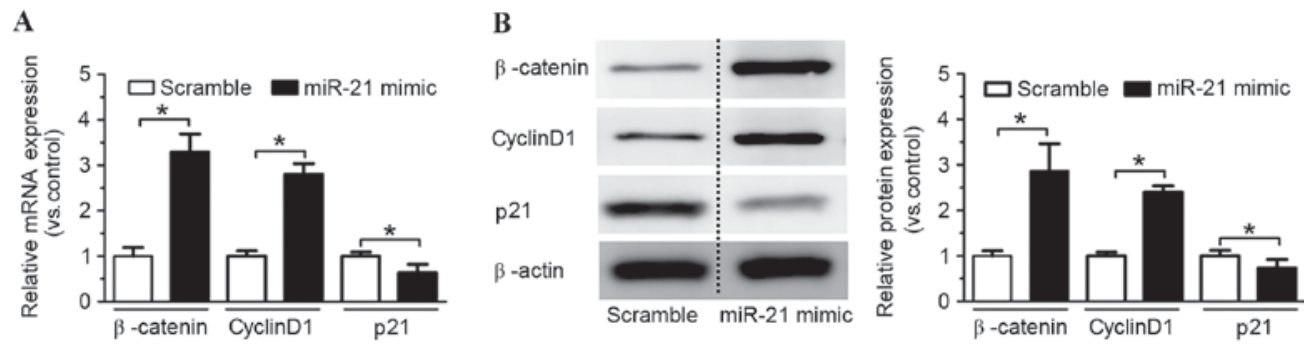

Figure 3. Effects of miR-21 overexpression on activation of the Wnt/ $\beta$-catenin signaling pathway in rat neural stem cells. (A) mRNA and (B) protein expression levels of selected genes involved in the Wnt/ $\beta$-catenin signaling pathway. Data were normalized to $\beta$-actin. Data are expressed as the mean \pm standard deviation $(\mathrm{n}=3) .{ }^{*} \mathrm{P}<0.05$ vs. scramble control group. miR-21, microRNA 21.

$\beta$-tubulin III expression and decreased GFAP expression compared with in the scramble control group $(\mathrm{P}<0.05$; Fig. 5).

\section{Discussion}

In the present study, miR-21 overexpression was demonstrated to promote rat NSC proliferation and neural differentiation, and to reduce differentiation to astrocytes, via the $\mathrm{Wnt} / \beta$-catenin signaling pathway. Furthermore, inhibition of the Wnt/ $\beta$-catenin signaling pathway impaired the miR-21-induced increase in NSC proliferation. Therefore, the present study revealed that miR-21 is involved in NSC proliferation and differentiation via the $\mathrm{Wnt} / \beta$-catenin signaling pathway.

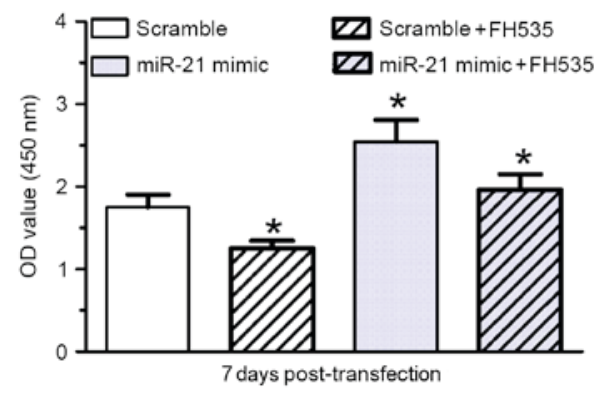

Figure 4. Inhibition of the Wnt/ $\beta$-catenin pathway with FH535 attenuates miR-21 overexpression-induced increased rat neural stem cell proliferation, analyzed by Cell Counting kit 8 assay. Data are expressed as the mean \pm standard deviation. " $\mathrm{P}<0.05$ vs. scramble control group or miR-21 mimic. miR-21, microRNA 21; OD, optical density. 


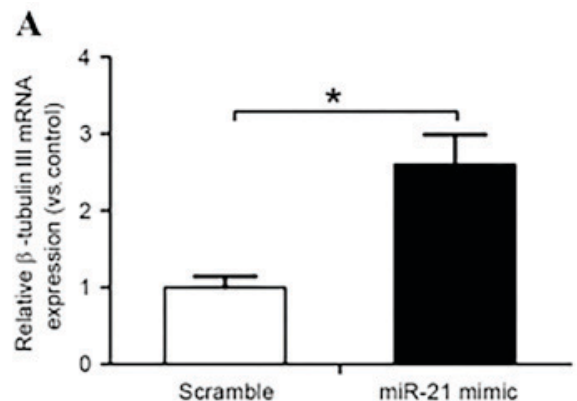

C
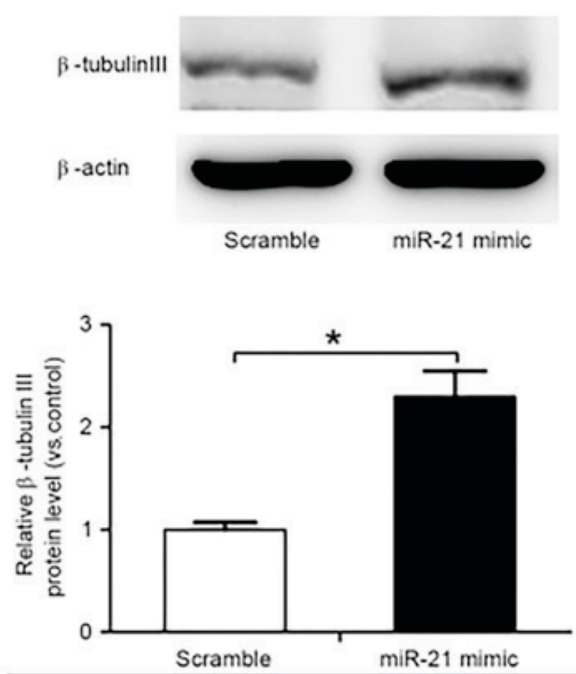

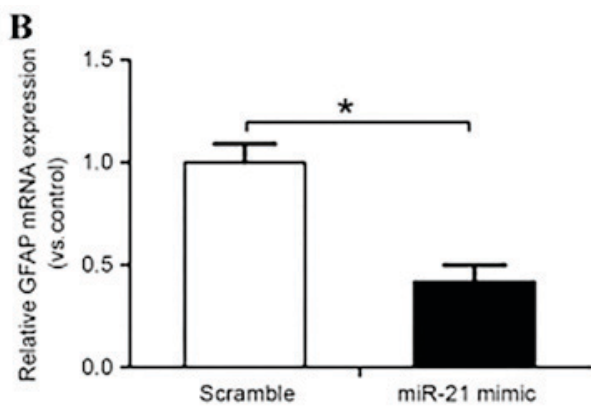

D
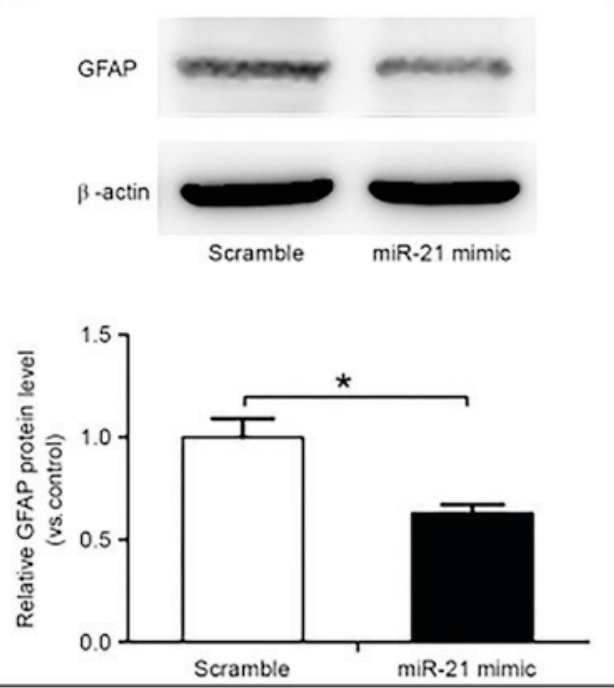

Figure 5. miR-21 promoted the differentiation of rat neural stem cells to neurons and inhibited differentiation to astrocytes. (A) $\beta$-tubulin III and (B) GFAP mRNA expression levels were detected by reverse transcription-quantitative polymerase chain reaction. (C) $\beta$-tubulin III and (D) GFAP protein expression levels were measured by western blotting. Data are expressed as the mean \pm standard deviation. ${ }^{*}$ P $<0.05$ vs. scramble control group. GFAP, glial fibrillary acidic protein; miR-21, microRNA 21.

The stimulatory effects of miR-21 on rat NSC proliferation have been confirmed in the present study. Compared with in the scramble control group, overexpression of miR-21 promoted NSC proliferation. In the present study cyclin D1 was upregulated and p21 was downregulated in response to miR-21 overexpression, as determined by RT-qPCR; p21 is a universal inhibitor of the cyclin/cyclin-dependent kinase (CDK) family (22). The cyclin D1 gene is a marker of cell cycle progression from $G_{1}$ to $S$ phase, and acts by binding to and activating CDKs (23). Furthermore, these findings were confirmed by western blot analysis. Cyclin D1 protein expression levels were upregulated, whereas p21 protein expression levels were downregulated in rat NSCs transfected with miR-21 mimic compared with the scramble controls. In addition, increased rat NSC proliferation was observed by EdU assay and CCK8 assay. These results are concordant with those of previous reports regarding other cell types. In lung carcinoma, the proliferative action of miR-21 was revealed to be associated with upregulation of cyclin D1 and downregulation of p21 (24). miR-21 is also considered to be a proliferative factor for human tubular epithelial cells (25), colorectal cancer cells (26) and colon cancer stem cells (27). The results from the present study, as well as from previous studies, confirm that miR-21 is involved in the regulation of NSC proliferation and differentiation.
The Wnt/ $\beta$-catenin signaling pathway is involved in cell proliferation and the differentiation of NSCs in the CNS during embryonic and adult neurogenesis (28). In mammals, the Wnt/ $\beta$-catenin signaling pathway is involved in the regulation of cellular growth, as well as numerous pathophysiological processes, including stroke and Alzheimer's disease (29-31). The present study revealed a potential association between miR-21 and the Wnt/ $\beta$-catenin signaling pathway. Overexpression of miR-21 was able to activate the Wnt/ $\beta$-catenin signaling pathway in rat NSCs, as determined by measuring the expression levels of the $\mathrm{Wnt} / \beta$-catenin pathway-associated markers $\beta$-catenin, cyclin D1 and 21 . $\beta$-catenin in the Wnt signaling pathway is a coactivator of $\mathrm{TCF} / \mathrm{lym}$ hoid enhancer factor (LEF)-dependent transcription, which is normally phosphorylated by glycogen synthase kinase $3 \beta$ and degraded quickly. In activation of the canonical Wnt pathway, increased levels of $\beta$-catenin are translocated to the nucleus. Nuclear $\beta$-catenin associates with the TCF/LEF transcription factor, and this complex subsequently binds to the promoter of Wnt-pathway genes, including cyclin D1, to activate transcription and promote proliferation. Increased levels of $\beta$-catenin indicate the activation of Wnt signaling (32). The results of the present study demonstrated that overexpression of miR-21 contributed to the increased $\beta$-catenin levels, and therefore activated the Wnt/ $\beta$-catenin pathway. Furthermore, 
a pharmacological inhibitor of $\beta$-catenin/TCF (FH535) was used to validate the involvement of the $\mathrm{Wnt} / \beta$-catenin signaling pathway in miR-21-induced rat NSC proliferation. Inhibition of the Wnt/ $\beta$-catenin pathway significantly attenuated the effects of miR-21 on rat NSC proliferation, as determined using a CCK8 assay. These findings confirmed that the Wnt/ $\beta$-catenin signaling pathway is required for miR-21-induced regulation of proliferation and differentiation of rat NSCs.

In conclusion, these data revealed the involvement of miR-21 in regulating proliferation and differentiation of rat NSCs. The present study also demonstrated that miR-21 regulated rat NSC proliferation and differentiation via the $\mathrm{Wnt} / \beta$-catenin signaling pathway. The present results suggested that miR-21 may have potential as a clinically useful tool and may represent a promising molecular target for the regulation of NSC proliferation.

\section{Acknowledgements}

The present study was supported by the Natural Science Fund of Henan Province (grant no. HN2013ZA042).

\section{References}

1. Aranha MM, Santos DM, Solá S, Steer CJ and Rodrigues CM: miR-34a regulates mouse neural stem cell differentiation. PLoS One 6: e21396, 2011.

2. Shi Y, Sun G,Zhao C and Stewart R: Neural stem cell self-renewal. Crit Rev Oncol Hematol 65: 43-53, 2008.

3. Koliatsos VE, Xu L and Cummings BJ: Stem cell therapies for traumatic brain injury. Regen Med 10: 917-920, 2015.

4. Bi YY, Feng DF and Pan DC: Stem/progenitor cells: A potential source of retina-specific cells for retinal repair. Neurosci Res 65: 215-221, 2009

5. Piltti KM, Avakian SN, Funes GM, Hu A, Uchida N, Anderson AJ and Cummings BJ: Transplantation dose alters the dynamics of human neural stem cell engraftment, proliferation and migration after spinal cord injury. Stem Cell Res 15: 341-353, 2015.

6. Lu H, Li M, Song T, Qian Y, Xiao X, Chen X, Zhang P, Feng X, Parker T and Liu Y: Retrovirus delivered neurotrophin-3 promotes survival, proliferation and neuronal differentiation of human fetal neural stem cells in vitro. Brain Res Bull 77: 158-164, 2008

7. Zhou XM, Sun JB, Yuan HP, Wu DL, Zhou XR, Sun DW, Li HY, Shao ZB and Zhang ZR: A rat model for studying neural stem cell transplantation. Acta Pharmacol Sin 30: 1496-1504, 2009.

8. Luo Y and Zhu D: Combinatorial control of transgene expression by hypoxia-responsive promoter and microrna regulation for neural stem cell-based cancer therapy. Biomed Res Int 2014: 751397, 2014

9. Lopez-Ramirez MA and Nicoli S: Role of miRNAs and epigenetics in neural stem cell fate determination. Epigenetics 9: 90-100, 2014

10. Rolando C and Taylor V: Neural stem cell of the hippocampus: Development, physiology regulation, and dysfunction in disease. Curr Top Dev Biol 107: 183-206, 2014.

11. Xia T, Li J, Cheng H, Zhang C and Zhang Y: Small-molecule regulators of MicroRNAs in biomedicine. Drug Dev Res 76 375-381, 2015.

12. Chen B, Li H, Zeng X, Yang P, Liu X, Zhao X and Liang S: Roles of microRNA on cancer cell metabolism. J Transl Med 10: 228, 2012.
13. Iwakawa HO and Tomari Y: The functions of MicroRNAs: mRNA decay and translational repression. Trends Cell Biol 25: 651-665, 2015.

14. Huang J, Zhang SY, Gao YM, Liu YF, Liu YB, Zhao ZG and Yang K: MicroRNAs as oncogenes or tumour suppressors in oesophageal cancer: Potential biomarkers and therapeutic targets. Cell Prolif 47: 277-286, 2014.

15. Fang S, Deng Y, Gu P and Fan X: MicroRNAs regulate bone development and regeneration. Int J Mol Sci 16: 8227-8253, 2015.

16. Li J, You T and Jing J: MiR-125b inhibits cell biological progression of Ewing's sarcoma by suppressing the PI3K/Akt signalling pathway. Cell Prolif 47: 152-160, 2014.

17. Li M, Yu M, Liu C, Zhu H, He X, Peng S and Hua J: miR-34c works downstream of p53 leading to dairy goat male germline stem-cell (mGSCs) apoptosis. Cell Prolif 46: 223-231, 2013.

18. Gangaraju VK and Lin H: MicroRNAs: Key regulators of stem cells. Nat Rev Mol Cell Biol 10: 116-125, 2009.

19. Choi E, Choi E and Hwang KC: MicroRNAs as novel regulators of stem cell fate. World J Stem Cells 5: 172-187, 2013.

20. Zhu W, Mao Y, Zhao Y, Zhou LF, Wang Y, Zhu JH, Zhu Y and Yang GY: Transplantation of vascular endothelial growth factor-transfected neural stem cells into the rat brain provides neuroprotection after transient focal cerebral ischemia. Neurosurgery 57: 325-333, 2005.

21. Livak KJ and Schmittgen TD: Analysis of relative gene expression data using real-time quantitative PCR and the 2(-Delta Delta C(T)) method. Methds 25: 402-408, 2001.

22. Xiong Y, Hannon GJ, Zhang H, Casso D, Kobayashi R and Beach D: p21 is a universal inhibitor of cyclin kinases. Nature 366: 701-704, 1993.

23. Sherr CJ: D-type cyclins. Trends Biochem Sci 20: 187-190, 1995.

24. Wu D, Shi M and Fan XD: Mechanism of miR-21 via Wnt/ $\beta$-catenin signaling pathway in human A549 lung cancer cells and Lewis lung carcinoma in mice. Asian Pac J Trop Med 8: 479-484, 2015.

25. Liu XJ,Hong Q, Wang Z, Yu YY,Zou X and Xu LH: MicroRNA21 promotes interstitial fibrosis via targeting DDAH1: A potential role in renal fibrosis. Mol Cell Biochem 411: 181-199, 2016.

26. Shi C, Yang Y, Xia Y, Okugawa Y, Yang J, Liang Y, Chen H, Zhang $\mathrm{P}$, Wang $\mathrm{F}$, Han $\mathrm{H}$, et al: Novel evidence for an oncogenic role of microRNA-21 in colitis-associated colorectal cancer. Gut 65: 1470-1481, 2016.

27. Yu Y, Nangia-Makker P, Farhana L, G Rajendra S, Levi E and Majumdar AP: miR-21 and miR-145 cooperation in regulation of colon cancer stem cells. Mol Cancer 14: 98, 2015.

28. Munji RN, Choe Y, Li G, Siegenthaler JA and Pleasure SJ: Wnt signaling regulates neuronal differentiation of cortical intermediate progenitors. J Neurosci 31: 1676-1687, 2011.

29. De Ferrari GV, Avila ME, Medina MA, Perez-Palma E, Bustos BI and Alarcon MA: Wnt/ $\beta$-catenin signaling in Alzheimer's disease. CNS Neurol Disord Drug Targets 13: 745-754, 2014

30. Mazur M,Bujak A,Matloka M,JanowskaS, Gunerka P,Bojarski L, Stanczak A, Klejman A, Bednarek A, Lamparska-Przybysz M and Wieczorek M: Cell-based assay for low-and high-scale screening of the Wnt $/ \beta$-catenin signaling modulators. Anal Biochem 475: 56-67, 2015.

31. Sun FL, Wang W, Zuo W, Xue JL, Xu JD, Ai HX, Zhang L, Wang XM and Ji XM: Promoting neurogenesis via Wnt/ $\beta$-catenin signaling pathway accounts for the neurorestorative effects of morroniside against cerebral ischemia injury. Eur J Pharmacol 738: 214-221, 2014.

32. Jamieson C, Sharma M and Henderson BR: Wnt signaling from membrane to nucleus: $\beta$-catenin caught in a loop. Int $\mathrm{J}$ Biochem Cell Biol 44: 847-850, 2012. 